\title{
Unveiling microbial activities along the halocline of Thetis, a deep-sea hypersaline anoxic basin
}

\author{
Maria G Pachiadaki ${ }^{1}$, Michail M Yakimov ${ }^{2}$, Violetta LaCono ${ }^{2}$, Edward Leadbetter ${ }^{1}$ and \\ Virginia Edgcomb ${ }^{1}$ \\ ${ }^{1}$ Woods Hole Oceanographic Institution, Woods Hole, MA, USA and ${ }^{2}$ CNR-Institute for Coastal Marine \\ Environment, Messina, Italy
}

\begin{abstract}
Deep-sea hypersaline anoxic basins (DHABs) in the Eastern Mediterranean Sea are considered some of the most hostile environments on Earth. Little is known about the biochemical adaptations of microorganisms living in these habitats. This first metatranscriptome analysis of DHAB samples provides significant insights into shifts in metabolic activities of microorganisms as physicochemical conditions change from deep Mediterranean sea water to brine. The analysis of Thetis DHAB interface indicates that sulfate reduction occurs in both the upper $(7.0-16.3 \%$ salinity) and lower (21.4-27.6\%) halocline, but that expression of dissimilatory sulfate reductase is reduced in the more hypersaline lower halocline. High dark-carbon assimilation rates in the upper interface coincided with high abundance of transcripts for ribulose 1,5-bisphosphate carboxylase affiliated to sulfuroxidizing bacteria. In the lower interface, increased expression of genes associated with methane metabolism and osmoregulation is noted. In addition, in this layer, nitrogenase transcripts affiliated to uncultivated putative methanotrophic archaea were detected, implying nitrogen fixation in this anoxic habitat, and providing evidence of linked carbon, nitrogen and sulfur cycles.
\end{abstract}

The ISME Journal (2014) 8, 2478-2489; doi:10.1038/ismej.2014.100; published online 20 June 2014

\section{Introduction}

Microorganisms exhibit an incredible diversity of physiologies and behaviors that allow them to exploit virtually all habitats on our planet. These habitats include many that seemed uninhabitable, such as those characterized by physicochemical parameters with values close to the limits known to support life. Deep-sea hypersaline anoxic basins (DHABs) are considered to be some of the most hostile environments because of their combination of extreme physicochemical features, including nearly saturated salt concentration and corresponding low water activity, high hydrostatic pressure, anoxia and high sulfide concentration. Typical DHABs of Eastern Mediterranean are $>3000 \mathrm{~m}$ below sea level; their origin is associated with the Miocene Messinian salinity crisis 5.59-5.33 million years ago. The genesis of the brine bodies on the sea floor of the Eastern Mediterranean Sea occurred 2000-176 000 years ago through either the de novo dissolution of buried Messinian evaporitic deposits or the ejection into seafloor depressions of ancient interstitial evaporated sea water entrapped in those deposits (Cita, 2006 and references therein). The

Correspondence: MG Pachiadaki or V Edgcomb, Woods Hole Oceanographic Institution, 266 Woods Hole Road, MS \#8, 02543 Woods Hole, MA, USA.

E-mail: mpachiadaki@whoi.edu or vedgcomb@whoi.edu

Received 4 February 2014; revised 30 April 2014; accepted 11 May 2014; published online 20 June 2014 extreme salinities (high densities) of DHABs act as a barrier to seawater mixing and salt diffusion, physically isolating them from other marine habitats, selecting for organisms adapted to multiple 'stressors' and probably preventing dispersal of those organisms. An interface layer, with a sharp oxycline, redoxcline and halocline, separates the hypersaline brine body and the oxygenated sea water.

Thus far, DHABs in the Mediterranean and Red Sea have provided exciting new insights into novel microbial diversity and have extended our knowledge of environmental factors that define the limits of life (Eder et al., 1999, 2001; Hallsworth et al., 2007). Studies of Discovery, L'Atalante, Urania, Thetis and Bannock basins revealed that DHAB interfaces harbor abundant and diverse microbial communities that include numerous novel candidate divisions that are more productive than most pelagic marine systems (see, for example, Sass et al., 2001; Van Der Wielen et al., 2005; Yakimov et al., 2007; Edgcomb et al., 2009; Stock et al., 2012). Functional analyses of these communities based on PCR amplification of key functional genes as well as activity measurements revealed sulfur cycling and methanogenesis to be dominant prokaryotic metabolic processes supporting life in DHABs and contributing to observed elevated biomass in DHAB haloclines (Daffonchio et al., 2006; Yakimov et al., 2007; Borin et al., 2009). Recent advances in RNA isolation, amplification and high-throughput 
sequencing make it possible to acquire millions of sequences of transcribed genes from microbial communities. Metatranscriptomics is a powerful tool for examining microbial community activities that enables inferences about the relative expression of diverse metabolic pathways based upon transcript abundance as a proxy for gene expression (Frias-Lopez et al., 2008; Stewart et al., 2011).

Thetis, discovered in 2008, is among the beststudied deep-sea hypersaline basins. Chemical analysis of the brine body shows it is almost nine times more saline than sea water, with an ion composition reflecting late-stage evaporites. Based on this, the Thetis brine is argued to originate from ancient trapped interstitial brines rather than dissolution of halite (La Cono et al., 2011). A pronounced stratification of both prokaryotic and eukaryotic communities was observed over the $\sim 2 \mathrm{~m}$ thick interface, as was a distinction between halocline and brine communities (La Cono et al., 2011; Stock et al., 2012). A recent metagenomics study of the Thetis brine and halocline (Ferrer et al., 2012) provided increased understanding of the metabolic potential of microbial communities of these habitats. However, $\left[{ }^{14} \mathrm{C}\right]$-bicarbonate assimilation rates and reverse transcriptase-PCR of a few functional genes in samples from the same Thetis habitats detected activities not suggested by the metagenome (La Cono et al., 2011), including the expression of ribulose 1,5-bisphosphate carboxylase. Such inconsistency points to the need for metatranscriptomic information on a wider range of metabolic activities for a better understanding of community processes.

To elucidate active metabolic pathways of microorganisms in the Thetis interface, we applied whole community metatranscriptomics to samples from two distinct positions along the halocline that differ in salinity and oxygen concentration. This study presents the first transcriptome analysis of microbiota from a DHAB halocline, and provides the first insights of expressed genes other than ribosomal RNA from the eukaryotic microbial community.

\section{Materials and methods}

\section{Sampling of Thetis halocline}

Sampling of Thetis $\left(34^{\circ} 40.158 \mathrm{~N}, 22^{\circ} 08.703 \mathrm{E}\right)$ was conducted from the $R / V$ Urania on 20 September 2012. The seawater/brine interface of Thetis is at a depth of $3258 \mathrm{~m}$ below sea level and the brine maximum thickness is $\sim 157 \mathrm{~m}$. The control sample (salinity $3.87 \%$ and oxygen $203 \mu \mathrm{moll}^{-1}$ ) from $2222 \mathrm{~m}$ depth $\left(36^{\circ} 29.565 \mathrm{~N}, 15^{\circ} 39.593 \mathrm{E}\right)$ was sampled on 28 September. Samples were collected using $12 \mathrm{l}$ Niskin bottles housed on a rosette (General Oceanics, Miami, FL, USA) equipped with a SBE- 911plus conductivity-temperature-depth sensor (Sea-Bird Electronics, Miami, FL, USA). Determination of oxygen concentration at selected depths was carried out using the Winkler method with an automatic end point detection burette (716 DNS Titrino, Metrohm AG, Herisau, Switzerland). Water from two distinct horizons (each representing a distinct range of salinity and oxygen between the top and bottom of each Niskin bottle) was collected from the Thetis interface; the upper interface (UI) layer corresponding to $7.0-16.3 \%$ salinity, and the lower (LI) layer with $21.4-27.6 \%$ salinity. Oxygen in the UI sample ranged from $9.5 \mu \mathrm{moll}^{-1}$ to undetectable, and remained undetectable in the lower sample. From each horizon, ca. $12 \mathrm{l}$ of water was collected on Durapore membranes $(47 \mathrm{~mm}, 0.65 \mu \mathrm{m}$, Millipore, Millford, MA, USA) under gentle pressure $\left(\sim 100 \mathrm{ml} \mathrm{min}^{-1}\right)$, using a peristaltic pump (Ecoline ISM 1079, Ismatec, Germany). The filters were stored in RNA Shield (Zymo Research, Orange, CA, USA) at $-80^{\circ} \mathrm{C}$ until analysis.

\section{RNA extractions}

Two replicate RNA extractions were processed per depth representing a total of $12 \mathrm{l}$ of water per horizon studied. The liquid RNA Shield and the filters were extracted separately. The RNA Shield was transferred to $50 \mathrm{ml}$ tubes and centrifuged at $4000 \mathrm{~g}$ for $3 \mathrm{~min}$ to remove particulates. An equal volume of $100 \%$ ethanol was added to the supernatant, mixed, transferred to an RNeasy Midi Kit (Qiagen, Hildesheim, Germany) column and processed following the manufacturer's recommendations. Contaminating DNA was removed by TURBO DNAse treatment (Life Technologies, Grand Island, NY, USA) according to the manufacturer's instructions. Total RNA was purified with the MEGA Clear Kit (Life Technologies) as directed and suspended in $100 \mu \mathrm{ld} \mathrm{d}_{2} \mathrm{O}$.

The filters were transferred into Lysing Matrix E tubes (MP Biomedicals, Solon, OH, USA). Then, $4 \mathrm{ml}$ of RNeasy Midi Kit Buffer RTL (Qiagen) was added, homogenized for $60 \mathrm{~s}$ at $4.0 \mathrm{~m} \mathrm{~s}^{-1}$ using a FastPrep-24 (MP Biomedicals) and centrifuged for $10 \mathrm{~min}$ at $4000 \mathrm{~g}$. Liquids were transferred to clean tubes, 1 volume of $70 \%$ ethanol was added to each tube and extracts were processed following the RNeasy kit instructions. Extractions were treated with DNase and purified with the MEGA Clear kit as described above. Fluid and filter extracts were combined for each sample and concentrated by ethanol precipitation. Absence of DNA was confirmed by 40 cycles of PCR using the general bacterial small subunit ribosomal RNA (SSU rRNA) gene primer $8 \mathrm{~F}$ and the universal primer $1492 \mathrm{R}$. A control deep-sea oxic sample was processed in a similar manner. More details about the control sample site and the processing of this sample are described in Edgcomb et al. (2014).

For each extraction of total RNA (two replicates per depth horizon), complementary DNA was synthesized using the Ovation RNA-Seq System V2 Kit (NuGEN Technologies, San Carlos, CA, USA) following the manufacturer's instructions. 
Finally, complementary DNAs were purified with the MinElute Reaction Cleanup Kit (Qiagen) and sent for paired-end sequencing. One lane of Illumina (San Diego, CA, USA) HiSeq $2 \times 100 \mathrm{bp}$ was requested for each sample (two replicates per lane). Sequences are deposited to the NCBI Short Read Archive (ID pending).

\section{Analysis of transcriptome data}

Forward and reverse reads were paired and filtered for quality control using CLC Genomics Workbench 5.0 (CLCBio, Cambridge, MA, USA) and a minimum quality score of 28, a minimum read length of $94 \mathrm{bp}$, allowing no sequences with ambigious nucleotides. The same platform was used to perform assembly of contigs and mapping of reads to contigs. The Rapid Analysis of Multiple Metagenomes with a Clustering and Annotation Pipeline (Weizhong, 2009) was used through the CAMERA platform (Sun et al., 2011) to assign contigs to clusters of orthologous gene (COG) families, gene ontologies (GO) and protein families (Pfam) using four translation tables (Supplementary Table S1) and an $e$-value of $10^{-5}$. Although the sequencing effort requested for each library was the same, different numbers of reads were recovered and survived quality trimming (Supplementary Table S2). To account for this variation (and the variation caused by difference in assembled reads and annotated contigs) and to compare abundances of transcripts among libraries, each library was normalized by expressing the number of annotated reads assigned to each function as a percentage of reads annotated to DNA-directed RNA polymerase subunit B (Stewart et al., 2011).

The degree of variation in gene expression profiles observed between each of the two replicates for each interface layer as well as the variation in expression profile between the two interface layers were assessed using R (R Development Core Team, 2008) version 3.0.2, and the DEGSeq package using a MAplot-based method with Random Sampling (Wang et al., 2009).

The rRNA prediction and annotation was performed by BLASTn (Altschul et al., 1990) against the SILVA 115 database (Quast et al., 2013) with an $e$ cutoff value of $10^{-10}$, whereas the taxonomic affiliation (binning) of the functional genes was done by BLASTx (Altschul et al., 1990).

\section{Results and discussion}

This is the first study using a metatranscriptome approach to examine microbial community activities within a DHAB habitat. The recently discovered Thetis DHAB was selected because a detailed description of the environmental setting, the diversity of its prokaryotes and eukaryotes, selected functional activities, and the genetic potential of the prokaryotic communities existed (La Cono et al., 2011; Ferrer et al., 2012; Stock et al., 2012). For the present study we examined the UI, from $7.0 \%$ to $16.3 \%$ salinity, and dissolved oxygen from $9.5 \mu \mathrm{moll}^{-1}$ to 0 , and the anoxic LI, with a salinity of $21.4 \%$ to $27.6 \%$. The topography of Thetis Basin and the depth profiles of oxygen, salinity and sulfide are shown in Supplementary Figure S1. Additional environmental parameters and cell counts are included in Supplementary Table S3.

Relative transcript abundance does not necessarily correlate directly with in situ activity levels, because potential biases can be introduced at the nucleic acid extraction step and during sample recovery using Niskin bottles that expose water samples and the microorganisms in them to significant environmental changes before filtration and preservation. Comparisons of metatranscriptomes for closely located samples (in time and space) subjected to the same potential biases can still provide valuable insights into ecologically relevant processes within the targeted habitats (Moran et al., 2013).

The taxonomic classification of partial gene transcripts is a major challenge for metatranscriptomic analyses. Several published taxonomic annotation tools were utilized, and given inconsistent annotations, we do not present taxonomic assignments for all metabolic gene transcripts. SSU rRNA reads were extracted from each data set to provide a separate taxonomic overview of the communities. Although our approach is free of PCR-related biases, relative abundances should be interpreted with caution because of differences in cell ribosome contents, potential biases at the level of RNA extraction and because our reverse transcription protocol intentionally biases against rRNA recovery. Consistency in the methodology applied to all samples, and good consistency observed between replicate samples for individual water features, enable us to compare results for the different water samples. The transcripts that showed significant $(P<0.05)$ difference expression levels between the UI and LI, as well as their normalized abundance in each one of the replicates, are reported in Supplementary Table S4.

\section{Taxonomic composition of microbial communities along the interface of Thetis lake} The archaeal groups dominating UI rRNA transcripts were Marine Group I (Thaumarchaeota) followed by Halobacteriales (Euryarchaeota), whereas the highest abundance of reads in the LI was affiliated with Methanomicrobiales (Euryarchaeota) (Figure 1). Within bacterial rRNA reads, Proteobacteria were the most abundant in both halocline layers. Signatures of Deltaproteobacteria and Gammaproteobacteria were the most abundant in UI libraries, whereas gammaproteobacterial signatures dominated the LI (Figure 1). We recovered signatures of Mediterranean Sea Brine Lake (MSBL) groups MSBL-2 and MSBL-8 (affiliated with 

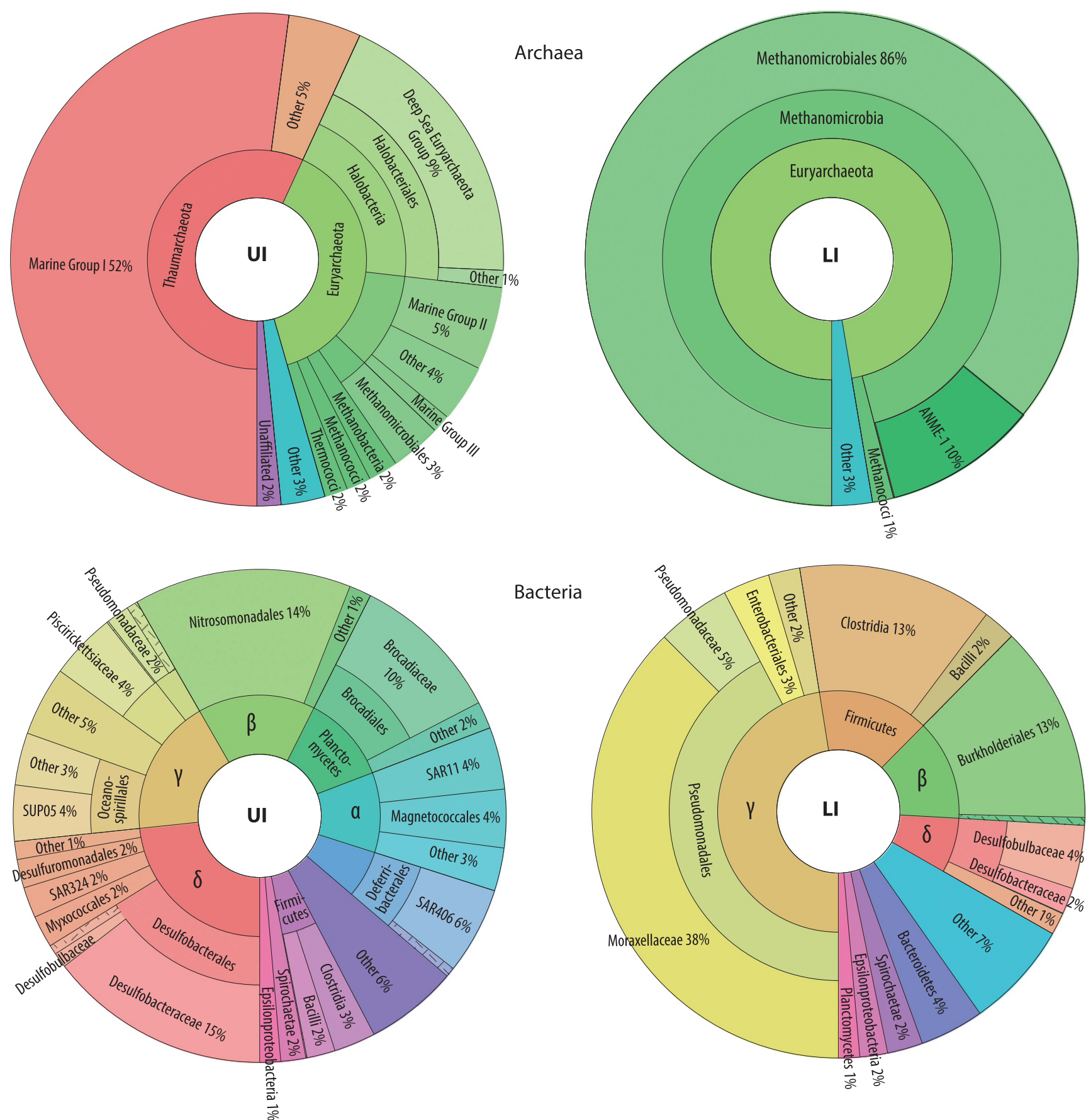

Bacteria

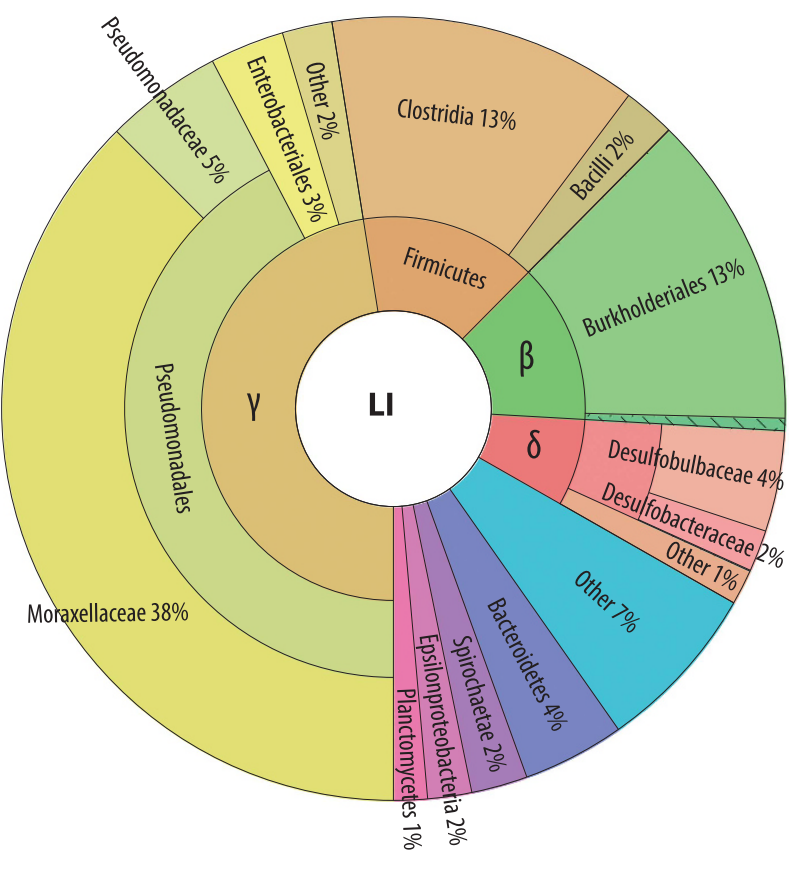

Figure 1 Relative abundance of rRNA reads of the major taxonomic groups of Archaea and Bacteria in the upper (UI) and lower (LI) interface of Thetis. 'Other' indicates taxa with relative abundance $<1 \%$.

Spirochaetes), MSBL-5 (affiliated with Chloroflexi), MSBL-6 (affiliated with Fusobacteria), MSBL-9 (affiliated with Planctomycetes) and MSBL-7 (affiliated with Deltaproteobacteria) from both interface layers, whereas MSBL-3 (affiliated with Lentisphaerae) signatures were only detected in the UI. Archaeal and bacterial rRNA profiles are generally consistent with previous studies (Yakimov et al., 2007; Borin et al., 2009; La Cono et al., 2011), although there were some differences, including the low abundance of epsilonproteobacterial and
Candidate Division KB1 signatures in our study. This may be attributed to differences in methodologies applied. A previous study of Thetis indicates that abundances (fluorescence in situ hybridization counts) of bacteria are higher than archaea in both UI and LI, 13.5 and 5.7 times, respectively (Supplementary Table S1).

The majority of unicellular eukaryotic reads in the UI were assigned to Ciliophora, whereas the LI was co-dominated by ciliate and fungal signatures (Figure 2). Euglenozoan, jakobid, rhizarian and 

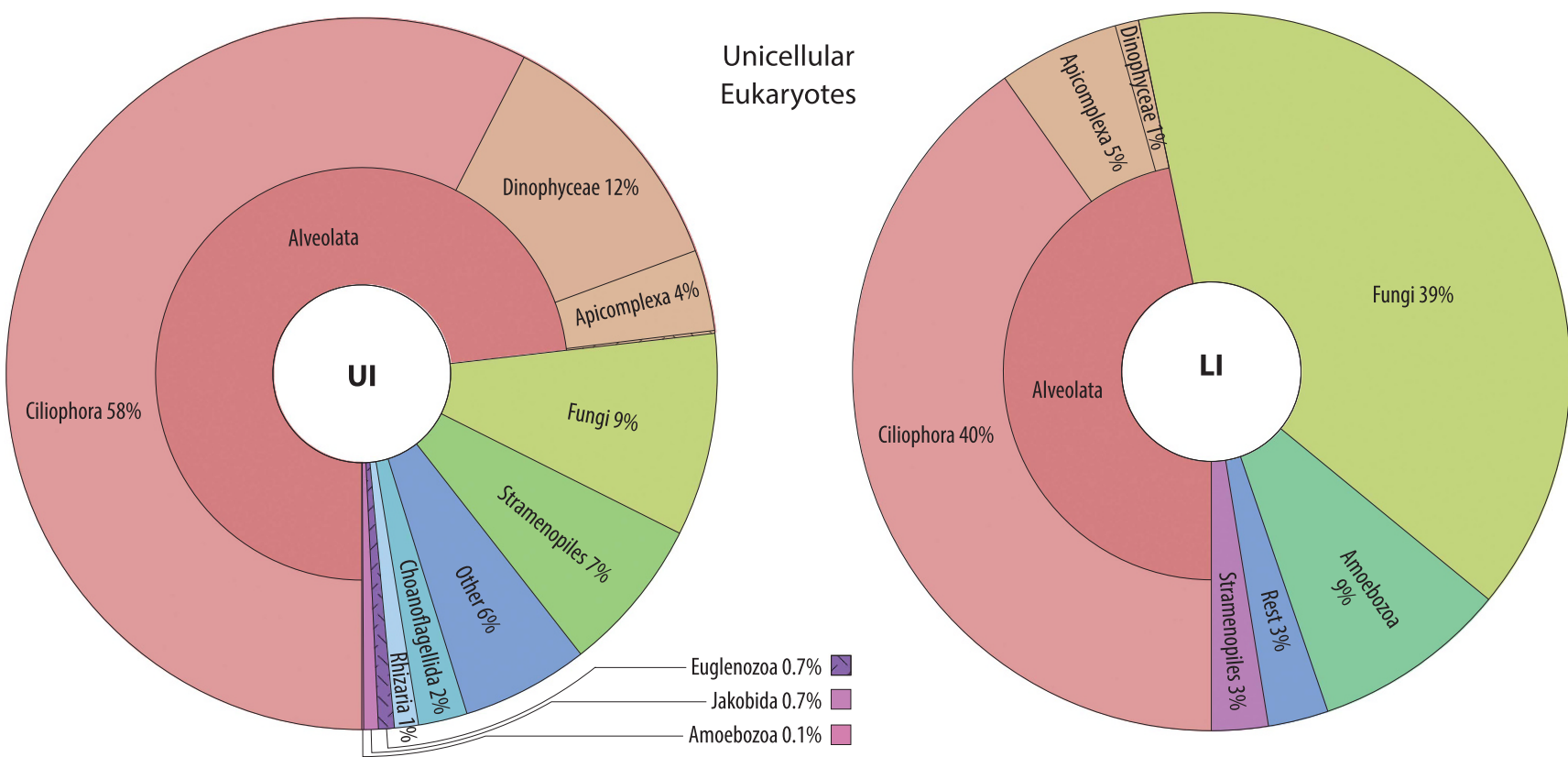

Figure 2 Relative abundance of the rRNA reads of the major taxonomic groups of the unicellular eukaryotes in the upper (UI) and lower (LI) interface of Thetis.

choanoflagellate signatures were only detected in the UI, indicating a decrease in diversity in the anoxic and more hypersaline layer. Other studies have found ciliates to dominate the eukaryotic microbial community in upper DHAB haloclines (Alexander et al., 2009; Edgcomb et al., 2009), but we observed a decrease in the relative abundance of ciliate rRNA as environmental conditions become more challenging in the LI, where Fungi appear equally abundant.

\section{Autotrophy $\left(\mathrm{CO}_{2}\right.$ fixation)}

In contrast to a previous metagenome study of Thetis interface (Ferrer et al., 2012), we detected transcripts for ribulose 1,5-bisphosphate carboxylase (RuBisCo), the indicative enzyme for the Calvin-Benson-Bassham cycle (CBB) in the UI. Transcripts for RuBisCo were also retrieved to a lesser extent from the LI (Figure 3a). UI transcripts were assigned to sulfur-oxidizing chemolithotrophs (Supplementary Table S5). Transcripts encoding RuBisCO were reported in the L'Atalante halocline, a DHAB with similar ionic concentrations (Yakimov et al., 2007), in the UI of Thetis (La Cono et al., 2011), and RuBisCO genes were detected in the athalassohaline Discovery basin (Van Der Wielen, 2006). Even though not predicted by the metagenome study of Thetis, the CBB cycle appears to be an important carbon fixation pathway in the UI. Elevated expression of RuBisCO coincides with high abundances of rRNA transcripts affiliated with Thiomicrospira and unassigned genera of the family Piscirickettsiaceae, as well as the SUP05 group within Gammaproteobacteria. RuBisCO-mediated
$\mathrm{CO}_{2}$ fixation is known among Thiomicrospira species (Dobrinski et al., 2005) and SUP05 (Jost et al., 2008; Walsh et al., 2009), and hence these taxa are likely among the key carbon fixers in the upper Thetis interface. The binning of RuBisCO contigs from the anoxic LI indicated they were derived from different (compared with the UI) groups. Only two groups were highly similar to known taxa, one to a methylotrophic, methanogenic archaeon and the second to an uncultivated bacterium from saline soils (Supplementary Table S5). Because carbon fixation activity was not detected in the LI (La Cono et al., 2011), a functioning RuBisCO involved in carbon fixation using nitrate is unlikely. These transcripts may be participating in adenosine 5'-monophosphate metabolism, a distinct function of classical RuBisCO (Sato et al., 2007). The CBB cycle is considered to be important in the mesopelagic and bathypelagic dark ocean (Swan et al., 2011). The high abundance of RuBisCO transcripts found in our deep-sea control data set supports this.

CO dehydrogenase/acetyl-CoA synthase catalyzes the reversible reduction of $\mathrm{CO}_{2}$ to $\mathrm{CO}$ and the subsequent synthesis/cleavage of acetyl-CoA (Pezacka and Harland, 1984) and is used as an indicator of autotrophic carbon fixation through the reductive acetyl-CoA (r acetyl CoA) pathway (Hügler and Sievert, 2011). In the control sample only the corrinoid Fe-S (delta) subunit was detected, whereas transcripts for all subunits of this enzyme complex were found in the UI and the LI. The majority of detected contigs in the UI were affiliated to bacteria, two to the metagenomes of uncultured Anaerobic Methane oxidizers Group 1 (ANME-1) and one to the methanogen Methanoregula formicica (Supplementary Table S5). 
a

CS

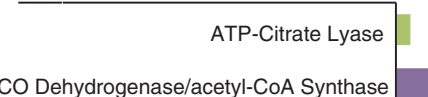

UI

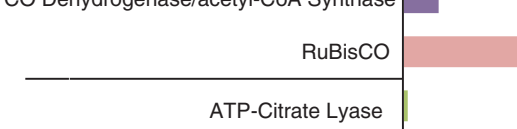

CO Dehydrogenase/acetyl-CoA Synthase

$$
- \text { RuBisCO }
$$

LI

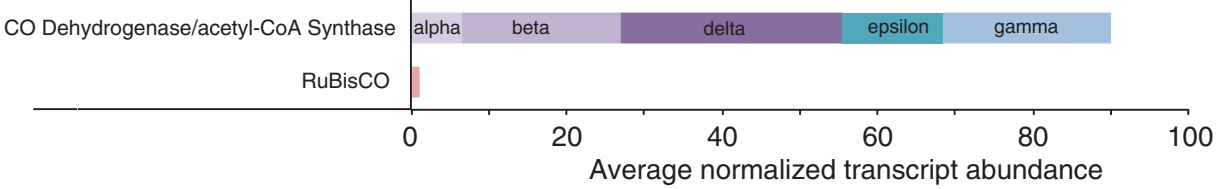

b

CS

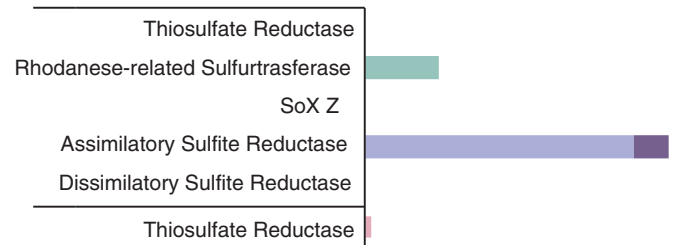

UI

Rhodanese-related Sulfurtrasferase

$$
\text { SoX Z }
$$

Assimilatory Sulfite Reductase

Dissimilatory Sulfite Reductase

Thiosulfate Reductase

Rhodanese-related Sulfurtrasferase

SoX Z

Assimilatory Sulfite Reductase

Dissimilatory Sulfite Reductase
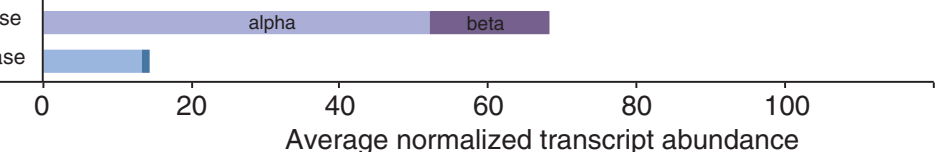

c

CS

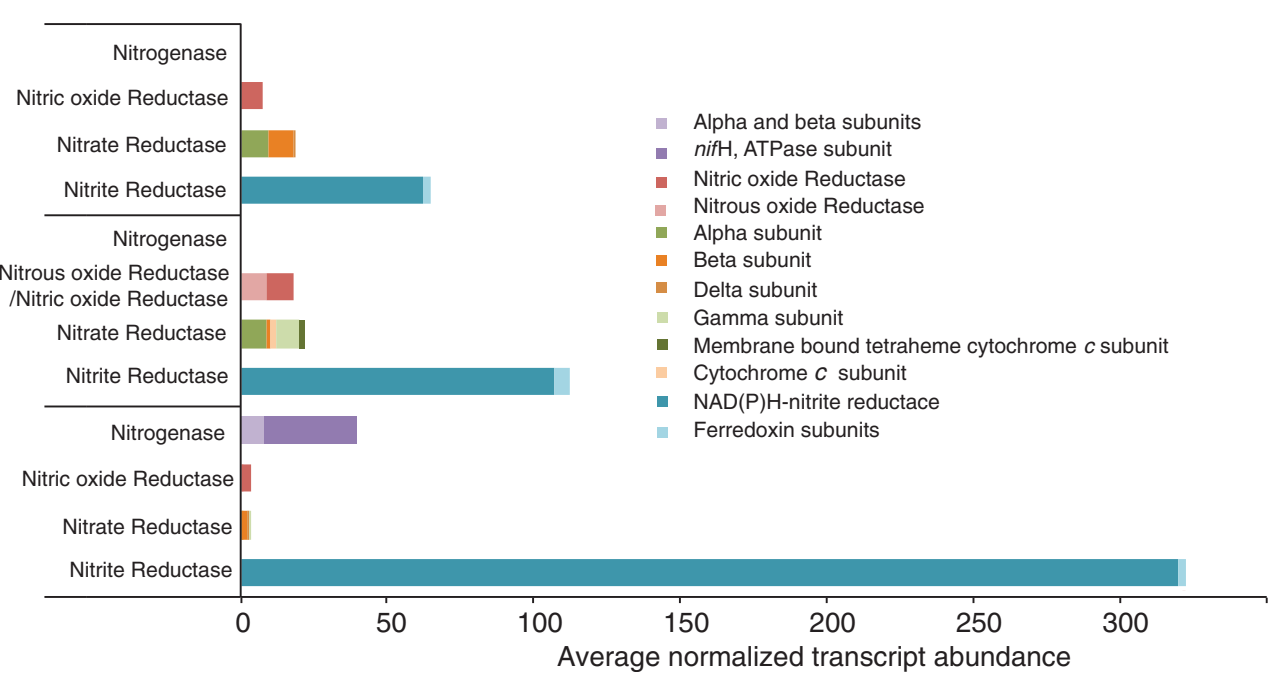

Figure 3 Average normalized transcript abundance of genes involved in the (a) carbon cycle (b) sulfur cycle and (c) nitrogen cycle. CS, control sample. For multi-unit enzymes in (a, b), each color represents a different subunit and the name of the subunit is indicated.

The bacterial contigs are closely related to taxa of the sulfate-reducing Desulfobacteracea family (Desulfospira, Desulfotignum, Desulfobacula and Desulfococcus). The CO dehydrogenase/acetyl-CoA synthase can be operating in either the oxidative heterotrophic or reductive CO-dehydrogenase pathway in these taxa (Kuever et al., 2001). In the LI, the majority of the CO dehydrogenase/acetyl-CoA synthase contigs were closely related (Supplementary Table S5) to the ANME-1 from the anaerobic oxidation of 
methane (AOM) system in Eel River Basin (Hallam et al., 2004).

Evidence for the presence of an active reductive tricarboxylic acid cycle was inconclusive. No contigs encoding the key enzyme 2-oxyglutarate synthase were detected, and only a few contigs for the other key enzyme, ATP-citrate lyase, were retrieved. No contigs for key enzymes of either 3-hydroxypropionate/4-hydroxybutyrate or 3- hydroxypropionate bicycle were found.

\section{Methanogenesis and $A O M$}

Although methanogenesis was thought to be a predominant process occurring in DHAB anoxic habitats (Borin et al., 2009), the Thetis metagenome did not suggest this (Ferrer et al., 2012). However, the transcriptome shows that the key enzyme of methanogenesis/AOM, methyl coenzyme $\mathrm{M}$ reductase, was highly expressed in both interface layers. This enzyme catalyzes the final reaction in biogenic methane production and its amino acid sequence is highly conserved among all methanogenic archaea (Ellermann et al., 1988; Grabarse et al., 2000). In AOM it is presumed to function in reverse (Hallam et al., 2003; Krüger et al., 2003; Wang et al., 2013). Transcripts for all subunits were significantly more abundant in the LI (Supplementary Figure S2), and were absent in the oxic control sample. Sequences exhibited high identity with methyl coenzyme M reductase sequences from the methanotrophic group ANME-1 (Supplementary Table S5). The absence of transcripts for coenzyme $\mathrm{F}_{420}$-dependent $\mathrm{N}_{5}, \mathrm{~N}_{10^{-}}$ methenyltetrahydromethanopterin dehydrogenase, which is lacking in ANME (Hallam et al., 2004), supports the hypothesis that ANMEs, performing AOM, are the key players here. The ANME-1 archaea were assumed to be obligatory methanotrophs, but recent studies provide evidence of co-occurrence of AOM and methanogenesis (Lloyd et al., 2011; Bertram et al., 2013; Wang et al., 2013). ANMEs were shown to assimilate inorganic carbon (Kellermann et al., 2012), but also to be capable of growing on acetate, pyruvate or butyrate using thiosulfate as an electron acceptor (Jagersma et al., 2012). The acetate-metabolizing potential in ANME2a was supported by the identification of the ion-motive electron transport complex Rnf that is independent of $\mathrm{H}_{2}$ (Wang et al., 2013). Previous studies of microbiota in this and other DHABs suggest autotrophy is precluded by high salinity in the LI and brine (Yakimov et al., 2007; La Cono et al., 2011); hence, the ANME community may function through a pathway that does not involve assimilation of inorganic carbon.

The sulfate concentration in Thetis brine is 10-fold higher than in sea water (La Cono et al., 2011). Although ANME-1 archaea have been found most frequently in sulfate-depleted environments (Yanagawa et al., 2011), they are known to be adapted to hypersalinity (Lloyd et al., 2006;
Maignien et al., 2013). This suggests salinity is a stronger selective force for this group than sulfate concentration.

\section{Metabolism of sulfur compounds}

High expression of sulfite reductase (assimilatory type) transcripts was observed in the LI, whereas in the UI expression appeared reduced relative to the LI and even relative to oxic deep-sea water (Figure 3b). Transcripts from all other enzymes in the assimilatory sulfate reduction pathway, ATP sulfurylase, 3'-phosphoadenosine 5'-phosphosulfate sulfotransferase (PAPS reductase) and adenosine-5'phosphosulfate-3'-phosphokinase (APS kinase), were detected in the LI. Evidence for dissimilatory sulfate reduction was apparent in both interface samples but not in the control deep-sea oxic sample. The abundance of dissimilatory sulfite reductase (desulfoviridin) transcripts was higher in the UI, in parallel with high abundance of rRNA transcripts from sulfate-reducing Deltaproteobacteria. The most represented family in the UI was Desulfobacteraceae that was reported previously in DHAB interfaces (Van Der Wielen and Heijs, 2007; Borin et al., 2009); their members are believed to be oxygen tolerant. In the LI, expression of dissimilatory sulfite reductase and rRNA of deltaproteobacterial sulfate reducers was reduced (Figure 3b). The Desulfobulbaceae appear to be the primary sulfate reducers in the LI. This family is known to be present in both interfaces and brines of DHABs (Van Der Wielen and Heijs, 2007; Borin et al., 2009) and other hypersaline environments (Ollivier et al., 1991; Kjeldsen et al., 2007).

Only low numbers of reads mapped to contigs encoding enzymes from the Sox (sulfur oxidation) enzyme system in interface samples, and these were not detected in the oxic control sample. Signatures of putative epsilonproteobacterial sulfide and sulfur oxidizers were in low abundance, but putative sulfide/sulfur-oxidizing Gammaproteobacteria were well represented, and were found to be involved in $\mathrm{CO}_{2}$ fixation through the CBB cycle in the UI. Sulfide/sulfur oxidation may be underestimated as this process is likely taking place in the uppermost layers of the interface where dissolved oxygen is available. The UI examined here corresponds to salinity $7.0-16.3 \%$ and oxygen content of 9.5 to $0 \mu \mathrm{mol} \mathrm{l}^{-1}$. We may have missed the peak expression of sulfide oxidation. Detected Epsilonproteobacteria were mostly affiliated with Arcobacter (Campylobacteriaceae) and Sulfurimonas and Sulfurovum (Helicobacteraceae). These taxa have been detected in both oxic and anoxic DHAB layers previously, and their potential role, apart from sulfur transformations, was thought to be associated with manganese reduction (Borin et al., 2009). Finally, evidence for nonreducing thiosulfate cleavage driven by RhoD rodanese-like thiosulfate:cyanide sulfurtransferases was detected in both interface layers as well as 
evidence for the reductive thiosulfate/thiosulfonate dismutation to sulfide by MopB-like thiosulfate reductases.

\section{Metabolism of nitrogen compounds}

The presence of transcripts for several different pathways within the nitrogen cycle was examined (Figure 3c). Transcripts indicative of denitrification and nitrous and nitric oxide reductases were detected in all samples. Expression levels in the LI were lower than in the UI and control sample. Similarly, transcripts for nitrate reductase subunits were less abundant in the LI compared with both UI and control samples. The abundance of NAD $(\mathrm{P}) \mathrm{H}-$ nitrite reductase transcripts showed an increase from the oxic deep sea to the LI. Finally, transcripts for nitrate/nitrate transporters and ammonia permeases were more abundant in the interface samples compared with the control.

No evidence for ammonia oxidation-apart from the high abundance of MG-1 Thaumarchaeota-or anaerobic ammonium oxidation (anammox) was found in either the UI or LI of Thetis. Anammox was shown to be active in the Black Sea oxycline (Kuypers et al., 2003) and in Atalante DHAB upper interface only up to $9.2 \%$ salinity (Borin et al., 2013), but no evidence of anammox activity was observed in the Thetis UI metatranscriptome. The redoxycline/halocline is so sharp that anammox might be active in an upper interface layer, but not captured.

Transcripts for the nitrogenase complex ( $\alpha$ and $\beta$ chains, as well as the ATPase component) were exclusively detected in the LI. This is the first time that evidence for nitrogen fixation activity in a DHAB habitat is reported. In ammonium-rich environments such as the DHAB interfaces and brines (Daffonchio et al., 2006), dinitrogen fixation is expected to be inhibited (Madigan et al., 2008), but some organisms are capable of maintaining high $\mathrm{N}_{2}$ fixation rates in the presence of a high level of ammonium. This was observed in isolates (Okoronkwo et al., 1989) and in natural populations from deep anoxic waters of the Baltic Sea (Farnelid et al., 2013). The binning of nitrogenase transcripts from the LI were to Archaea, including methanogens and ANME. Methanogens are considered to be among the nitrogen-fixing free-living anaerobes (Madigan et al., 2008), and recently it was demonstrated that deep-sea ANME from ammoniumreplete sediments fix $\mathrm{N}_{2}$ and share the products of nitrogen fixation with sulfate-reducing bacterial partners (Dekas et al., 2009; Offre et al., 2013). We do not have an explanation for why methanogens/ ANME compensate for the energetic burden of diazotrophy in an ammonium-rich environment. In the LI, the high relative abundance of transcripts for ammonia permease indicates that other pathways of nitrogen assimilation co-occur. It is possible that LI prokaryotes are fixing nitrogen and assimilating ammonia in order to synthesize osmoprotectants via glutamine synthase and glutamate synthase pathways.

\section{Osmolytes}

Two strategies are used by microorganisms for coping with the osmotic stresses associated with low water activity in hypersaline environments. They can achieve osmotic stability using inorganic ions such as $\mathrm{KCl}$ or, more commonly, microorganisms can produce and accumulate low-molecular-mass osmolytes. These include organic compounds such as amino acids and derivatives, alcohols, polyols (for example, sugars, manitol, arabitol, glycerol) and their derivatives, betaines and thetines and ectoines that protect enzymes and macromolecular structures from inactivation, inhibition and denaturation under low water activity (Grant, 2004; Stan-Lotter and Fendrihan, 2013).

These first insights into strategies for osmoprotection in DHABs via osmolyte synthesis show that differential expression of putative osmoprotectants, such as glutamine, glutamate, proline, ectoine and 2-sulfotrehalose, was detected in halocline vs control samples (Supplementary Figure S3). Glutamine synthetase transcript abundance was similar in the deep-sea oxic water sample and in the UI, but showed an increase in the LI, suggesting this mechanism becomes increasingly important as salinity increases. The same pattern was observed for proline biosynthesis pathway transcripts. Significantly higher abundance of proline biosynthesis pathway transcripts for pyrroline-5-carboxylate reductase, glutamate 5-kinase and glutamate-5-semialdehyde dehydrogenase were observed in the LI vs UI. Transcripts for choline dehydrogenase, a key enzyme for glycine betaine biosynthesis, and for lysine 2,3-aminomutase showed a similar pattern. Consistent with synthesis of putative osmoprotectants, abundances of transcripts for osmoregulator transporters, including the choline-glycine betaine transporter, were higher in the LI. Glutamate synthase expression was higher in both interface layers compared with the control sample. Among sugar and polyol compounds that are also used as osmoprotectants, transcripts of trehalose-6-phosphate synthase were 10 times more abundant in the LI than in the UI and the control. Similarly, myoinositol-1-phosphate synthase was expressed at levels 20 times higher in LI relative to UI. These findings indicate that multiple adaptive mechanisms for coping with high salinity are utilized by microorganisms inhabiting the UI and LI, and that expression of associated genes increases with salinity along the halocline.

Ion transporters

Transcripts for several types of $\mathrm{Na}^{+}$and $\mathrm{K}^{+}$ transporters were recovered that can provide insight 
about strategies for osmoprotection via regulation of ion concentrations. Most were more abundant in the interface layers relative to the oxic deep-sea water. Nha-type $\mathrm{Na}^{+} / \mathrm{H}^{+}$antiporter and $\mathrm{K}^{+}$transporter transcripts were more expressed in the UI relative to the control (Supplementary Figure S4A). In both LI and UI, expression of the $\mathrm{NhaC} \mathrm{Na}^{+} / \mathrm{H}^{+}$antiporter was similar, but a substantial increase in expression of the NhaP and Mnh systems was observed in the $\mathrm{LI}$, as was expression of all $\mathrm{K}^{+}$transport systems, particularly Kdp (Supplementary Figures S4A and B). This implies that LI communities adapt to hypersalinity using the 'salt-in', rather than 'salt-out' adaptation strategy, consistent with generally accepted statements about energetic constraints associated with life at high salinity (Oren, 2013). Ion concentrations are two to four times higher than in sea water in the UI, and seven- to eight-fold higher in the LI. Different groups of organisms are probably using different osmoregulation mechanisms, but at the community level both strategies, 'salt-in' and osmolyte synthesis, seem to be active in DHAB interfaces. Finally, transcripts of Rnf were enriched in the LI ( $\sim 150$ times higher than in sea water). The presence of sodium-motive ferredoxin:NAD oxidoreductase (Rnf) subunits may indirectly indicate the presence of organisms capable of completely relying on a sodium ion potential for energetic reactions (Biegel et al., 2011; Poehlein et al., 2012). The Rnf membrane-associated electrogenic antiporter is apparently involved in generating sodium ion gradients. This gradient may be further used for ATP synthesis under highly reduced conditions at redox below $-320 \mathrm{mV}$.

\section{Eukaryotic signatures}

As we did not base our metatranscriptome libraries on exclusively polyA-selected transcripts, it is difficult to unequivocally assign many metabolic gene transcripts to eukaryotes as many have close homologs in prokaryotes. We therefore refrain in this study from reporting on specific eukaryotic metabolic activities. To assess their relative activity in the Thetis UI and LI, transcripts for two structural proteins were used as indicators. Actin and tubulin proteins from eukaryotes have only weak amino acid similarity compared with the prokaryotic cytoskeletal proteins (Desai and Mitchison, 1998). The abundances of transcripts were more than four times higher in the UI compared with the control site, whereas there was a significant drop of the abundances in the LI (Supplementary Figure S5). This is in accordance with a previous study (Edgcomb et al., 2011b) reporting high numbers of unicellular eukaryotes in the UI layers of DHABs. The LI, a high-saline totally anoxic habitat, appears to be more challenging for eukaryotes.

Extremely halophilic heterotrophic protists that are distinct from marine or freshwater forms have been observed in solar salterns with salinity of $29 \%$
(Cho et al., 2008). A diversity of other heterotrophic protists were isolated from nearly saturated brines $(\geqslant 30 \%$ salinity) that could not grow at salinities of $<7.5 \%$ (Cho et al., 2008; Park et al., 2006; Park et al., 2007, 2009). Based on SSU rRNA gene transcripts recovered within our metatranscriptome libraries, the eukaryotic community appears to be dominated by ciliates and dinoflagellates in the UI, and by ciliates and fungi in the LI (Figure 2). Previous studies of DHABs, including Thetis, based on SSU rRNA and microscopic observations have detected heterotrophic protists (particularly ciliates, dinoflagellates and fungi) in up to $36 \%$ salinity (see, for example, Alexander et al., 2009; Edgcomb et al., 2009; Stock et al., 2012). Phagotrophic protists are known to be successful along pelagic oxyclines where prokaryotes are abundant (see, for example, Behnke et al., 2006; Edgcomb et al., 2011a) and hence it follows that they are also successful within these haloclines. Fungi are active remineralizers of organic material, and given the known accumulation of organic detritus at these haloclines, and their known presence in other types of hypersaline habitats such as solar salterns (for example, Cantrell et al., 2006), it is not surprising that their signatures dominate in the lower halocline.

Recovery of transcribed genes from a slightly broader taxonomic representation of eukaryotes in the UI vs LI is consistent with previous findings of rich and diverse communities in moderately saline systems (Hauer and Rogerson, 2005). Signatures from apicomplexans and stramenopiles were also found in the UI and LI, and unique to the UI, we detected signatures from jakobids, euglenozoans and choanoflagellates. All these groups have been detected in previous SSU RNA- and DNA-based molecular surveys of DHABs (for overview see Edgcomb and Bernhard, 2013). Further elucidation of the specific adaptive strategies and ecological roles and impacts on nutrient cycling of these protists is a fascinating avenue for future research.

\section{Conclusion}

In this first metatranscriptomic study of a deep-sea redoxcline/halocline, we find that microbial communities of the upper-hypoxic to anoxichalocline are shaped by transformations of sulfur compounds and inorganic carbon assimilation, occurring primarily through the CBB cycle. In the lower anoxic, more saline habitat, it is likely that AOM rather than methanogenesis may be the dominant process. Transcripts for dinitrogen fixation were detected in the same habitat, where ammonium concentrations are considered prohibitive for such a process. Laboratory incubations of ammonium-replete $\mathrm{CH}_{4}$ seep sediments demonstrated that methanotrophic archaea are capable of dinitrogen fixation (Dekas et al., 2009). This finding, 
although it requires confirmation, suggests new sources of fixed nitrogen in nontraditional, 'unexpected' marine habitats, linking the function of carbon, nitrogen and sulfur cycles.

\section{Conflict of Interest}

The authors declare no conflict of interest.

\section{Acknowledgements}

We thank the captains and crews of the $R / V$ Urania for their hard work to assure the success of our sampling objectives, Craig Taylor (WHOI), Gina La Spada, Francesco Smedile, Gioacchino Ruggeri and the rest of the Marine Molecular Microbiology and Biotechnology group at CNR, Messina, for assistance with sampling and Karen Casciotti and Samantha Joye for helpful discussions on the $\mathrm{N}$ cycle. Cruise participation was partly funded by the Deutsche Forschungsgemeinschaft (DFG) STO414/10-1 to Thorsten Stoeck. This research was funded by NSF OCE-0849578 to VE.

\section{Author contributions}

MGP and VE designed the study and collected the samples. MGP conducted data analysis and manuscript preparation with input from EL and VE. VL performed chemical analyses reported here. MMY was the chief scientist on the $R / V$ Urania cruise, and assisted with sample collection and manuscript preparation.

\section{References}

Alexander E, Stock A, Breiner HW, Behnke A, Bunge J, Yakimov MM et al. (2009). Microbial eukaryotes in the hypersaline anoxic L'Atalante deep-sea basin. Environ Microbiol 11: 360-381.

Altschul SF, Gish W, Miller W, Myers EW, Lipman DJ. (1990). Basic local alignment search tool. J Mol Biol 215: 403-410.

Behnke A, Bunge J, Barger K, Breiner H-W, Alla V, Stoeck T. (2006). Microeukaryote community patterns along an $\mathrm{O}_{2} / \mathrm{H}_{2} \mathrm{~S}$ gradient in a supersulfidic anoxic fjord (Framvaren, Norway). Appl Environmen Microbiol 72: $3626-3636$.

Bertram S, Blumenberg M, Michaelis W, Siegert M, Krüger M, Seifert R. (2013). Methanogenic capabilities of ANME-archaea deduced from ${ }^{13} \mathrm{C}$-labelling approaches. Environ Microbiol 15: 2384-2393.

Biegel E, Schmidt S, González J, Müller V. (2011). Biochemistry, evolution and physiological function of the Rnf complex, a novel ion-motive electron transport complex in prokaryotes. Cell Mol Life Sci 68: $613-634$.

Borin S, Brusetti L, Mapelli F, D’Auria G, Brusa T, Marzorati $\mathrm{M}$ et al. (2009). Sulfur cycling and methanogenesis primarily drive microbial colonization of the highly sulfidic Urania deep hypersaline basin. Proc Natl Acad Sci USA 106: 9151-9156.
Borin S, Mapelli F, Rolli E, Song B, Tobias C, Schmid M et al. (2013). Anammox bacterial populations in deep marine hypersaline gradient systems. Extremophiles 17: 289-299.

Cantrell SA, Casillas-Martinez L, Molina M. (2006). Characterization of fungi from hypersaline environments of solar salterns using morphological and molecular techniques. Mycol Res 110: 962-970.

Cho BC, Park JS, Xu K, Choi JK. (2008). Morphology and molecular phylogeny of Trimyema koreanum n. sp., a Ciliate from the hypersaline water of a solar saltern. J Eukaryot Microbiol 55: 417-426.

Cita MB. (2006). Exhumation of Messinian evaporites in the deep-sea and creation of deep anoxic brine-filled collapsed basins. Sediment Geol 188-189: 357-378.

Daffonchio D, Borin S, Brusa T, Brusetti L, Van Der Wielen PWJJ, Bolhuis $\mathrm{H}$ et al. (2006). Stratified prokaryote network in the oxic-anoxic transition of a deep-sea halocline. Nature 440: 203-207.

Dekas AE, Poretsky RS, Orphan VJ. (2009). Deep-sea Archaea fix and share nitrogen in methane-consuming microbial consortia. Science 326: 422-426.

Desai A, Mitchison TJ. (1998). Tubulin and FtsZ structures: functional and therapeutic implications. BioEssays 20: 523-527.

Dobrinski KP, Longo DL, Scott KM. (2005). The carbon-concentrating mechanism of the hydrothermal vent chemolithoautotroph Thiomicrospira crunogena. J Bacteriol 187: 5761-5766.

Eder W, Ludwig W, Huber R. (1999). Novel 16S rRNA gene sequences retrieved from highly saline brine sediments of Kebrit Deep, Red Sea. Arch Microbiol 172: 213-218.

Eder W, Jahnke LL, Schmidt M, Huber R. (2001). Microbial diversity of the brine-seawater Interface of the Kebrit Deep, Red Sea, studied via 16S rRNA gene sequences and cultivation methods. Appl Environ Microbiol 67: 3077-3085.

Edgcomb V, Orsi W, Leslin C, Epstein SS, Bunge J, Jeon S et al. (2009). Protistan community patterns within the brine and halocline of deep hypersaline anoxic basins in the eastern Mediterranean Sea. Extremophiles 13: 151-167.

Edgcomb V, Orsi W, Taylor GT, Vdacny P, Taylor C, Suarez P et al. (2011a). Accessing marine protists from the anoxic Cariaco Basin. ISME J 5: 1237-1241.

Edgcomb VP, Orsi W, Breiner HW, Stock A, Filker S, Yakimov MM et al. (2011b). Novel active kinetoplastids associated with hypersaline anoxic basins in the Eastern Mediterranean deep-sea. Deep-Sea Res PT I 58: 1040-1048.

Edgcomb V, Bernhard J. (2013). Heterotrophic protists in hypersaline microbial mats and deep hypersaline basin water columns. Life 3: 346-362.

Edgcomb VP, Taylor C, Pachiadaki MG, Hongo S, Engstrom I, Yakimov MM. (2014). Comparison of niskin vs. in situ approaches for analysis of gene expression in deep Mediterranean Sea water samples. Deep Sea Res II (in press).

Ellermann J, Hedderich R, Böcher R, Thauer RK. (1988). The final step in methane formation. Eur J Biochem 172: 669-677.

Farnelid H, Bentzon-Tilia M, Andersson AF, Bertilsson S, Jost G, Labrenz $M$ et al. (2013). Active nitrogen-fixing heterotrophic bacteria at and below the chemocline of the central Baltic Sea. ISME J 7: 1413-1423. 
Ferrer M, Werner J, Chernikova TN, Bargiela R, Fernández L, La Cono V et al. (2012). Unveiling microbial life in the new deep-sea hypersaline Lake Thetis. Part II: a metagenomic study. Environ Microbiol 14: 268-281.

Frias-Lopez J, Shi Y, Tyson GW, Coleman ML, Schuster SC, Chisholm SW et al. (2008). Microbial community gene expression in ocean surface waters. Proc Natl Acad Sci USA 105: 3805-3810.

Grabarse W, Mahlert F, Shima S, Thauer RK, Ermler U. (2000). Comparison of three methyl-coenzyme M reductases from phylogenetically distant organisms: unusual amino acid modification, conservation and adaptation. J Mol Biol 303: 329-344.

Grant WD. (2004). Life at low water activity. Philos T Roy Soc B 359: 1249-1267.

Hallam SJ, Girguis PR, Preston CM, Richardson PM, DeLong EF. (2003). Identification of methyl coenzyme $\mathrm{M}$ reductase A (mcrA) genes associated with methaneoxidizing archaea. Appl Environ Microbiol 69: 5483-5491.

Hallam SJ, Putnam N, Preston CM, Detter JC, Rokhsar D, Richardson PM et al. (2004). Reverse methanogenesis: testing the hypothesis with environmental genomics. Science 305: 1457-1462.

Hallsworth JE, Yakimov MM, Golyshin PN, Gillion JLM, D’Auria G, De Lima Alves F et al. (2007). Limits of life in $\mathrm{MgCl}_{2}$-containing environments: chaotropicity defines the window. Environ Microbiol 9: 801-813.

Hauer G, Rogerson A. (2005). Heterotrophic protozoa from hypersaline environments. In: Gunde-Cimerman N, Oren A, Plemenitaš A (eds). Adaptation to Life at High Salt Concentrations in Archaea, Bacteria, and Eukarya. Springer: The Netherlands, pp 519-539.

Hügler M, Sievert SM. (2011). Beyond the Calvin cycle: autotrophic carbon fixation in the ocean. Ann Rev Mar Sci 3: 261-289.

Jagersma CG, Meulepas RJW, Timmers PHA, Szperl A, Lens PNL, Stams AJM. (2012). Enrichment of ANME-1 from Eckernförde Bay sediment on thiosulfate, methane and short-chain fatty acids. J Biotechnol 157: 482-489.

Jost G, Zubkov MV, Yakushev E, Labrenz M, Jürgens K. (2008). High abundance and dark $\mathrm{CO}_{2}$ fixation of chemolithoautotrophic prokaryotes in anoxic waters of the Baltic Sea. Limnol Oceanogr 53: 14-22.

Kellermann MY, Wegener G, Elvert M, Yoshinaga MY, Lin Y-S, Holler T et al. (2012). Autotrophy as a predominant mode of carbon fixation in anaerobic methane-oxidizing microbial communities. Proc Natl Acad Sci USA 109: 19321-19326.

Kjeldsen KU, Loy A, Jakobsen TF, Thomsen TR, Wagner M, Ingvorsen K. (2007). Diversity of sulfate-reducing bacteria from an extreme hypersaline sediment, Great Salt Lake (Utah). FEMS Microbiol Ecol 60: 287-298.

Krüger M, Meyerdierks A, Glöckner FO, Amann R, Widdel F, Kube $\mathrm{M}$ et al. (2003). A conspicuous nickel protein in microbial mats that oxidize methane anaerobically. Nature 426: 878-881.

Kuever J, Könneke M, Galushko A, Drzyzga O. (2001). Reclassification of Desulfobacterium phenolicum as Desulfobacula phenolica comb. nov. and description of strain SaxT as Desulfotignum balticum gen. nov., sp. nov. Int J Syst Evol Micr 51: 171-177.

Kuypers MMM, Sliekers AO, Lavik G, Schmid M, Jorgensen BB, Kuenen JG et al. (2003). Anaerobic ammonium oxidation by anammox bacteria in the Black Sea. Nature 422: 608-611.

La Cono V, Smedile F, Bortoluzzi G, Arcadi E, Maimone G, Messina E et al. (2011). Unveiling microbial life in new deep-sea hypersaline Lake Thetis. Part I: Prokaryotes and environmental settings. Environ Microbiol 13: 2250-2268.

Lloyd KG, Lapham L, Teske A. (2006). An anaerobic methane-oxidizing community of ANME-1b archaea in hypersaline gulf of Mexico sediments. Appl Environ Microbiol 72: 7218-7230.

Lloyd KG, Alperin MJ, Teske A. (2011). Environmental evidence for net methane production and oxidation in putative ANaerobic MEthanotrophic (ANME) archaea. Environ Microbiol 13: 2548-2564.

Madigan MT, Martinko JM, Parker J. (2008). Brock Biology of Microorganisms, 12th edn Pearson.

Maignien L, Parkes RJ, Cragg B, Niemann H, Knittel K, Coulon S et al. (2013). Anaerobic oxidation of methane in hypersaline cold seep sediments. FEMS Microbiol Ecol 83: 214-231.

Moran MA, Satinsky B, Gifford SM, Luo H, Rivers A, Chan L-K et al. (2013). Sizing up metatranscriptomics. ISME J 7: 237-243.

Offre P, Spang A, Schleper C. (2013). Archaea in biogeochemical cycles. Ann Rev Microbiol 67: $437-457$.

Okoronkwo N, Hovel C, Eskew DL. (1989). Evaluation of nitrogen fixation by different strains of the AzollaAnabaena symbiosis in the presence of a high level of ammonium. Biol Fert Soils 7: 275-278.

Ollivier B, Hatchikian CE, Prensier G, Guezennec J, Garcia JL. (1991). Desulfohalobium retbaense gen. nov., sp. nov., a halophilic sulfate-reducing bacterium from sediments of a hypersaline lake in Senegal. Int J Syst Bacteriol 41: 74-81.

Oren A. (2013). Life at high salt concentrations. In: Rosenberg E, DeLong E, Lory S, Stackebrandt E, Thompson F (eds) The Prokaryotes. Springer: Berlin Heidelberg, pp 421-440.

Park JS, Simpson AGB, Brown S, Cho BC. (2009). Ultrastructure and molecular phylogeny of two Heterolobosean Amoebae, Euplaesiobystra hypersalinica gen. et sp. nov. and Tulamoeba peronaphora gen. et sp. nov., isolated from an extremely hypersaline habitat. Protist 160: 265-283.

Park JS, Simpson AGB, Lee WJ, Cho BC. (2007). Ultrastructure and phylogenetic placement within heterolobosea of the previously unclassified, extremely halophilic heterotrophic Flagellate Pleurostomum flabellatum (Ruinen 1938). Protist 158: 397-413.

Pezacka E, Harland GW. (1984). Role of carbon monoxide dehydrogenase in the autotrophic pathway used by acetogenic bacteria. Proc Natl Acad Sci USA 81: 6261-6265.

Poehlein A, Schmidt S, Kaster A-K, Goenrich M, Vollmers J, Thürmer A et al. (2012). An ancient pathway combining carbon dioxide fixation with the generation and utilization of a sodium ion gradient for ATP synthesis. PLoS One 7: e33439.

Quast C, Pruesse E, Yilmaz P, Gerken J, Schweer T, Yarza P et al. (2013). The SILVA ribosomal RNA gene database project: improved data processing and web-based tools. Nucleic Acids Res 41: D590-D596.

$\mathrm{R}$ Development Core Team (2008). R: A Language and Environment for Statistical ComputingVienna, Austria. 
Sass AM, Sass H, Coolen MJL, Cypionka H, Overmann J. (2001). Microbial communities in the chemocline of a hypersaline deep-sea basin (Urania Basin, Mediterranean Sea). Appl Environ Microbiol 67: 5392-5402.

Sato T, Atomi H, Imanaka T. (2007). Archaeal type III RuBisCOs function in a pathway for AMP metabolism. Science 315: 1003-1006.

Stan-Lotter H, Fendrihan S. (2013). Survival strategies of halophilic oligotrophic and desiccation resistant prokaryotes. Polyextremophiles. Springer: The Netherlands, pp 233-248.

Stewart F, Dmytrenko O, DeLong E, Cavanaugh C. (2011). Metatranscriptomic analysis of sulfur oxidation genes in the endosymbiont of Solemya velum. Front Microbiol 2: 134.

Stock A, Breiner H-W, Pachiadaki M, Edgcomb V, Filker S, Cono V et al. (2012). Microbial eukaryote life in the new hypersaline deep-sea basin Thetis. Extremophiles 16: $21-34$.

Sun S, Chen J, Li W, Altintas I, Lin A, Peltier S et al. (2011). Community cyberinfrastructure for Advanced Microbial Ecology Research and Analysis: the CAMERA resource. Nucleic Acids Res 39: D546-D551.

Swan BK, Martinez-Garcia M, Preston CM, Sczyrba A, Woyke T, Lamy D et al. (2011). Potential for chemolithoautotrophy among ubiquitous bacteria lineages in the dark ocean. Science 333: 1296-1300.

Van Der Wielen PWJJ. (2006). Diversity of ribulose1,5-bisphosphate carboxylase/oxygenase large-subunit genes in the $\mathrm{MgCl}_{2}$-dominated deep hypersaline anoxic basin discovery. FEMS Microbiol Let 259: 326-331.

Van der Wielen PWJJ, Bolhuis H, Borin S, Daffonchio D, Corselli C, Giuliano L et al. (2005). The enigma of prokaryotic life in deep hypersaline anoxic basins Science 307: 121-123.

Van Der Wielen PWJJ, Heijs SK. (2007). Sulfate-reducing prokaryotic communities in two deep hypersaline anoxic basins in the Eastern Mediterranean deep sea. Environ Microbiol 9: 1335-1340.

Walsh DA, Zaikova E, Howes CG, Song YC, Wright JJ, Tringe SG et al. (2009). Metagenome of a versatile chemolithoautotroph from expanding oceanic dead zones. Science 326: 578-582.

Wang F-P, Zhang Y, Chen Y, He Y, Qi J, Hinrichs K-U et al. (2013). Methanotrophic archaea possessing diverging methane-oxidizing and electron-transporting pathways. ISME J 8: 1069-1078.

Wang L, Feng Z, Wang X, Wang X, Zhang X. (2009). DEGseq: an R package for identifying differentially expressed genes from RNA-seq data. Bioinformatics 26: 136-148.

Weizhong L. (2009). Analysis and comparison of very large metagenomes with fast clustering and functional annotation. BMC Bioinformatics 10: 359.

Yakimov MM, La Cono V, Denaro R, D’Auria G, Decembrini F, Timmis KN et al. (2007). Primary producing prokaryotic communities of brine, interface and seawater above the halocline of deep anoxic lake L'Atalante, Eastern Mediterranean Sea. ISME J 1: 743-755.

Yanagawa K, Sunamura M, Lever MA, Morono Y, Hiruta A, Ishizaki $\mathrm{O}$ et al. (2011). Niche separation of methanotrophic archaea (ANME-1 and -2) in methane-seep sediments of the Eastern Japan Sea offshore Joetsu. Geomicrobiol J 28: 118-129.

Supplementary Information accompanies this paper on The ISME Journal website (http://www.nature.com/ismej) 